\title{
Key principles and methods for studying the endocytosis of biological and nanoparticle therapeutics
}

\author{
Joshua J. Rennick $\circledast^{1,2}$, Angus P. R. Johnston $\oplus^{1,2} \llbracket$ and Robert G. Parton $\left(^{2,3} \llbracket\right.$
}

Endocytosis is a critical step in the process by which many therapeutic nanomedicines reach their intracellular targets. Our understanding of cellular uptake mechanisms has developed substantially in the past five years. However, these advances in cell biology have not fully translated to the nanoscience and therapeutics literature. Misconceptions surrounding the role of different endocytic pathways and how to study these pathways are hindering progress in developing improved nanoparticle therapies. Here, we summarize the latest insights into cellular uptake mechanisms and pathways. We highlight limitations of current systems to study endocytosis, particularly problems with non-specific inhibitors. We also summarize alternative genetic approaches to robustly probe these pathways and discuss the need to understand how cells endocytose particles in vivo. We hope that this critical assessment of the current methods used in studying nanoparticle uptake will guide future studies at the interface of cell biology and nanomedicine.

E ngineering nanoparticles to deliver drugs has the potential to improve cancer therapy, vaccination and treatment of genetic disorders. The key advantages of nanoparticle delivery lie in the potential to reduce side effects by targeting specific cells, protecting delicate therapeutics from premature degradation, improving the solubility of hard-to-deliver drugs and providing sustained and controlled release of drugs. While a number of barriers must be overcome to achieve targeted nanoparticle drug delivery, ultimately in most cases the ability for a drug to have a therapeutic effect at its site of action is governed by the capacity of the nanoparticle to enter the cell.

Internalization pathways are not ubiquitous. Phagocytosis and macropinocytosis, for example, may not be a feature of all cell types. However, it is less well recognized that other commonly studied internalization pathways are also limited to certain cells. When investigating nanoparticle uptake, it is crucial to understand if the pathways being studied are actually present in the cells. Determining the mechanism of uptake can give important information about efficiency of delivery, therapeutic activity and translation to other cells or in vivo studies. However, reporting an uptake mechanism for the sake of it and without reference to the biological relevance adds little to our knowledge.

There are two main routes of entry into the cell: direct fusion with the plasma membrane or endocytosis. Direct fusion to the plasma membrane is exploited by some encapsulated viruses ${ }^{1}$, and a number of interesting nanoparticle systems have been engineered to exploit this pathway ${ }^{2,3}$. However, the principal route of entry of nanoparticles into the cell is via endocytosis. The importance of understanding endocytosis is highlighted by the effect nanoparticle targeting has on tumour regression. In vivo targeting of nanoparticles to tumours typically results in $<1 \%$ of the total dose accumulating at the tumour site ${ }^{4}$. However, compared with non-targeted nanoparticles, the tumour regression for targeted systems is substantially greater than the increased accumulation. This suggests that uptake of nanoparticles by tumour cells is the driving force behind the increased activity of targeted nanoparticles. Understanding endocytosis is also important for engineered nanoparticles that can avoid clearance by the mononuclear phagocytic system (MPS) ${ }^{5}$, phagocytic immune cells that form the first line of defence in the body against foreign invaders. A major hindrance to nanoparticle therapies is the rapid clearance of nanoparticles by the MPS, which results in accumulation in the liver and spleen. This accumulation results in a loss of active therapeutic from the circulation and can result in significant toxic side effects if the nanoparticle carries a cytotoxic payload. A critical step in avoiding clearance by the MPS is avoiding nanoparticle uptake by these cells.

An understanding of endocytosis can also benefit the development of different types of therapeutic strategy, independent of nanoparticle uptake. For example, a recent study demonstrated that the antitumour effects of anti-EGFR (epidermal growth factor receptor) antibodies can be potentiated by inhibition of specific endocytic pathways, leading to enhanced antibody-dependent cellular cytotoxicity mediated by natural killer cells ${ }^{6}$.

The role of endocytosis is also important for naturally occurring nanoparticles, such as viruses and exosomes. The rise of viruses such as SARS-CoV-2 highlights the importance of understanding uptake mechanisms, as this understanding can help to inform potential antiviral treatments. Exosome signalling plays an important role in normal growth and development, but also plays a key role in the progression of diseases such as cancer. Exosomes can also be exploited for their therapeutic potential, and are currently being used for short interfering RNA (siRNA), protein and small-molecule drug delivery ${ }^{7}$. As with synthetic nanoparticles, the ability of exosomes to carry out these functions is governed by the ability of a target cell to internalize the exosome and its cargo.

While cellular uptake plays a critical role in the efficiency of therapeutic delivery, the analysis of internalization is often hampered by an outdated understanding of the mechanisms that drive cellular uptake. In this Review we will highlight the latest advances in our

'Monash Institute of Pharmaceutical Sciences, Monash University, Parkville, Victoria, Australia. ${ }^{2}$ ARC Centre of Excellence in Convergent Bio-Nano Science and Technology, Brisbane, Queensland, Australia. ${ }^{3}$ Institute for Molecular Bioscience and Centre for Microscopy and Microanalysis, The University of Queensland, Brisbane, Queensland, Australia. $\bigotimes_{e}$-mail: angus.johnston@monash.edu; r.parton@imb.uq.edu.au 


\section{Box 1 | Key points to consider in nanoparticle uptake experiments}

1. What endocytic pathways exist in the cell culture system being used and in the target cells of interest in vivo?

2. Does efficient uptake in a cultured cell equate to efficient uptake in vivo?

3. Are inhibitors specific for a single pathway and are they non-toxic (are there positive and negative controls with pathway-specific markers)?

4. Can genetic perturbation be used to define the endocytic pathway(s) being used?

5. How does a nanoparticle of interest pass from the bloodstream to the target tissue?

understanding of the cellular mechanisms of internalization and critically assess the current techniques that can be used to determine the pathways involved. We also provide guidelines for future experiments assessing nanoparticle uptake (Box 1).

\section{Pathways of endocytosis}

Understanding the mechanisms by which nanoparticles can be internalized into cells is important for a number of reasons. First, the physical properties (for example size) of the nanoparticles govern the mechanisms that are able to mediate the uptake of the particle. Second, the rate of nanoparticle uptake is largely dependent on the internalization mechanism. Finally, not all cells possess the same internalization machinery; therefore, understanding the mechanisms of uptake can inform which cells will be likely to internalize nanoparticles that are engineered to target specific pathways. A poor understanding of internalization mechanisms can lead to poorly engineered nanoparticles that have suboptimal therapeutic performance and can lead to unexpected results when studying nanoparticles with different cell lines, or when translating nanoparticles from in vitro to in vivo studies. The same nanoparticle may also be internalized by different mechanisms in different cell types ${ }^{8,9}$, again emphasizing the need to understand endocytic pathways in the model cell studied in culture and within the target cells of interest in vivo.

Before considering the relevance of different endocytic pathways for nanoparticles to enter the cell and reach their intracellular targets, a brief summary of the current view of endocytosis is required. This is particularly important in view of recent major developments in our understanding of the endocytic pathways in vertebrate cells, particularly those that do not use clathrin. For more extensive reviews of this area the reader is referred to recent articles ${ }^{10,11}$.

A consensus is now developing for five major types of endocytosis: (1) clathrin-coated pit-mediated endocytosis (CME; clathrin and dynamin dependent), (2) fast endophilin-mediated endocytosis (FEME, a clathrin-independent but dynamindependent pathway for rapid ligand-driven endocytosis of specific membrane proteins), (3) clathrin-independent carrier (CLIC)/ glycosylphosphatidylinositol-anchored protein enriched early endocytic compartment (GEEC) endocytosis (clathrin and dynamin independent), (4) macropinocytosis and (5) phagocytosis (Fig. 1 and Box 2). Caveolae represent a sixth pathway, which in theory can also contribute to endocytic uptake. While caveolae can bud from the plasma membrane, few if any cargoes are dependent on caveolae for their uptake ${ }^{12}$ and endocytosis by the FEME and CLIC/GEEC pathways may be confused with the caveola pathway due to poor specificity of endocytosis inhibitors (Tools to study mechanisms of uptake and Table 1).

These endocytic pathways will be briefly considered here as a framework for understanding the uptake of nanoparticles. Model cargoes for each pathway are highlighted, but no cargo is shown for the caveolin pathway due to the limited evidence of protein cargo that is specifically internalized by this pathway. A common feature of these pathways is that the majority of cargoes they internalize are trafficked to the early endosome. Cargo can be recycled back to the cell surface or onwards to the late endosome and subsequently to the lysosome. It should also be appreciated that we are presenting a simplified view of the pathways as a working model for classifying and defining the different endocytic mechanisms. The study of endocytosis is an actively evolving field, so it is important for nanomaterial researchers to remain up to date with the latest advances in cellular understanding. Clathrin, for example, is a defining feature of CME but can also be involved in the uptake of large particles through a mechanism that is distinct from the classical CME pathway. Boundaries between CLIC/GEEC endocytosis and macropinocytosis can also be unclear, and the two pathways may share common components ${ }^{13}$. There is also clearly cross-talk between pathways: for example, perturbation of the levels of caveolar components can affect the CLIC/GEEC pathway ${ }^{14}$. Finally, inhibition of one pathway can modulate another and so compensate for the lack of that pathway. For example, inhibition of dynamin using a temperature-sensitive mutant causes rapid upregulation of dynamin-independent endocytosis ${ }^{15}$. This is a crucial consideration in studies using inhibitors of specific pathways to define uptake mechanisms and emphasizes the need for multiple techniques.

Clathrin-mediated endocytosis, CME. The clathrin-dependent pathway of endocytosis is the best understood endocytic route. It occurs in all mammalian cells and is the principal route for cells to obtain nutrients, for example facilitating the uptake of iron (via transferrin) and cholesterol (via low-density lipoproteins). CME is sometimes referred to as receptor-mediated endocytosis; however, this term is outdated and misleading, as clathrin-independent internalization mechanisms can also involve specific receptor-ligand interactions (for example the FEME pathway) ${ }^{16}$. Clathrin-coated pits occupy $0.5-2 \%$ of the cell surface ${ }^{17}$. Receptor clustering or phosphorylation within the cytoplasmic domain of surface membrane proteins recruits adaptin proteins and initiates a cascade of low-affinity protein-protein and protein-lipid interactions (particularly with phosphatidylinositol 4,5-bisphosphate $\left.\left(\operatorname{PtdIns}(4,5) \mathrm{P}_{2}\right)\right)$, which lead to the formation of a clathrin-coated pit. This is a highly dynamic and cooperative system in which a multitude of interactions form a pit within $30-120 \mathrm{~s}$ of ligand binding ${ }^{18}$. The pit rapidly invaginates to form a clathrin-coated vesicle, which pinches off the plasma membrane through the activity of dynamin, a large mechanical GTPase. Clathrin-coated vesicles have an average diameter of $\sim 100 \mathrm{~nm}\left(\right.$ ref. ${ }^{18}$ ), representing the upper size limit of nanoparticles that can be internalized via this pathway.

Bacteria and large particles up to $1 \mu \mathrm{m}$ in diameter have been shown to co-opt clathrin and form actin-rich pedestals to facilitate their uptake ${ }^{19}$. It has also been proposed that rod-like viruses and nanoparticles, longer than the diameter of the typical clathrin-coated vesicle, can be internalized by CME through the actin elongation of the clathrin-coated $\mathrm{pit}^{20}$. The requirement for actin recruitment can slow the endocytic process, leading to altered internalization kinetics compared with conventional CME.

The distinct morphology of the clathrin-coated pit, systems to effectively and specifically perturb the pathway, and well characterized cargoes that are completely dependent on this pathway for uptake have facilitated the extensive characterization of this ubiquitous and constitutive pathway.

Clathrin-independent/dynamin-dependent endocytosis, FEME. FEME has recently emerged as an important pathway for rapid endocytosis of specific transmembrane receptors, important in growth factor signalling and in cell migration ${ }^{16}$. Cargoes for FEME 


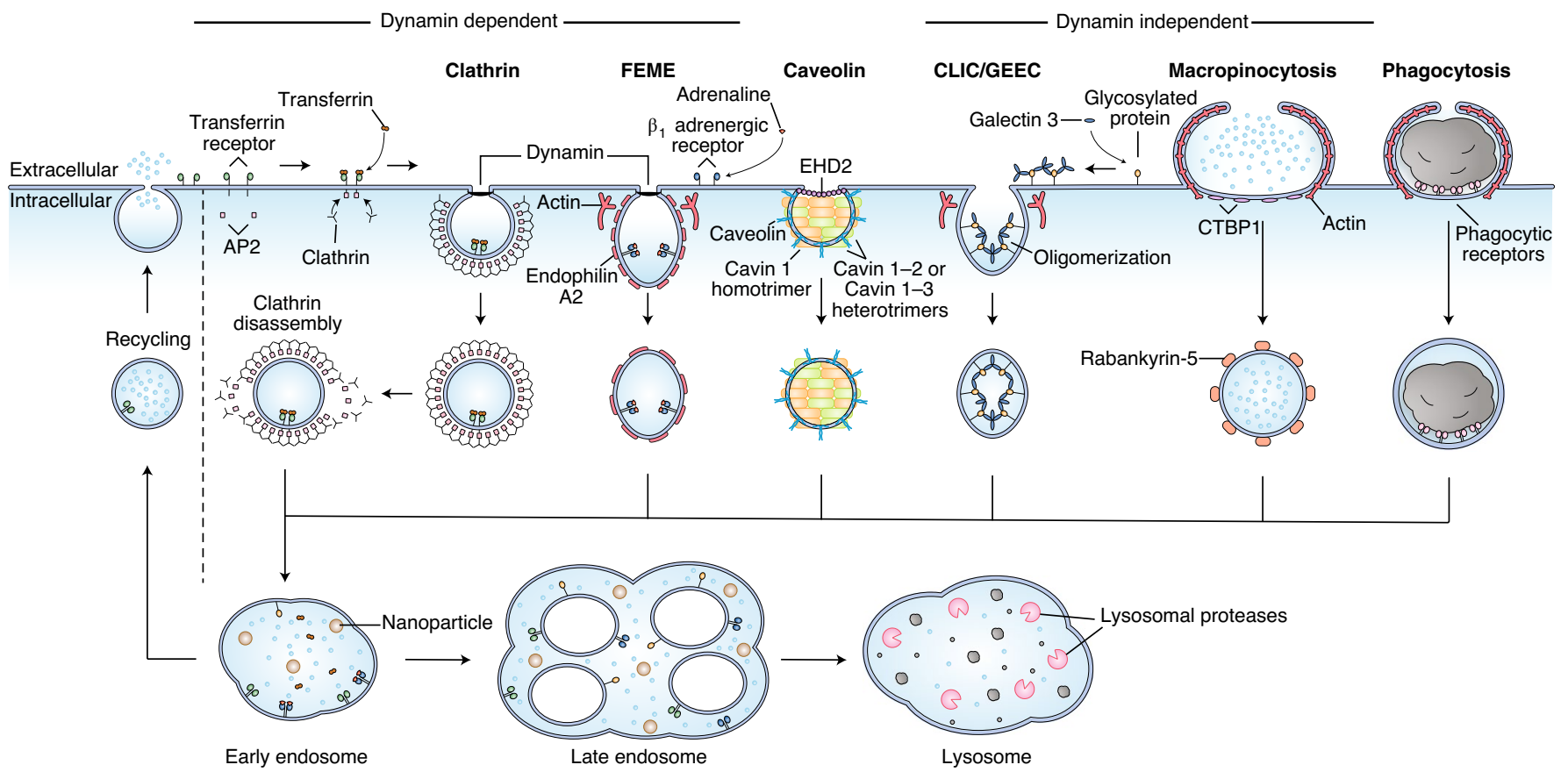

Fig. 1 | Overview of the primary mechanisms of uptake into cells. CME is driven by the adaptor complex, AP2, that recruits clathrin to cytosolic receptor domains, initiating the formation of a clathrin-coated pit. FEME is triggered by ligand-receptor interactions and regulated by endophilin A2 recruitment and actin polymerization. Both CME and FEME require dynamin to facilitate scission from the membrane and involve interactions of the intracellular domains of cell surface receptors with cytoplasmic machinery. Formation of caveolae is dependent on caveolin and cavin proteins. EHD2 stabilizes the neck of caveolae to regulate endocytosis. CLIC/GEEC endocytosis appears to be a constitutive process (that is, a continuous endocytic uptake mechanism independent of receptor-ligand interactions) and is clathrin and dynamin independent. Extracellular galectin proteins (not shown) cocluster glycoproteins and glycolipids to drive carrier formation and cargo incorporation. Macropinocytosis is generally a stimulated pathway involving uptake of large volumes of fluid. CLIC/GEEC endocytosis and macropinocytosis are controlled by actin dynamics and different BAR domain proteins with macropinosome fission from the surface also influenced by C-terminal-binding protein 1 (CTBP1). Phagocytosis occurs after a binding event at the cell surface triggers actin polymerization and a vesicle forms tightly around the bound material. After internalization, pathways merge into early endosomes before undergoing sorting, where material may be sent back to the surface or onwards to the late endosome and lysosome.

include G-protein-coupled receptors, such as $\beta_{1}$-adrenergic, dopaminergic and acetylcholine receptors, as well as the IL-2 receptor and growth factor receptors (EGFR, hepatocyte growth factor receptor (HGFR)).

The FEME pathway has a number of unique features that distinguish it from other pathways. First, the pathway is clathrin independent but dynamin dependent. Second, the formation of endocytic carriers is induced upon ligand binding to specific receptors. FEME relies on interactions between the $\mathrm{SH} 3$ domain of endophilin and cognate receptors (for example G-protein-coupled receptors) or indirect association through intermediate proteins such as CIN85 and $\mathrm{Cbl}$ (for EGFR and HGFR). These interactions stabilize endophilin localized to the leading edge of migrating cells through the PtdIns $(3,4) \mathrm{P}_{2}$-binding protein lamellipodin. Formation of FEME carriers is extremely rapid $(<10 \mathrm{~s})$, is dependent on pre-enrichment of endophilin at the membrane and leads to the formation of tubular carriers, which are $60-80 \mathrm{~nm}$ in diameter and several hundred nanometres in length ${ }^{16}$.

Clathrin-independent/dynamin-independentendocytosis, CLIC/ GEEC. CLICs are involved in a distinct endocytic pathway, independent of dynamin or clathrin, which has been shown to be a high-capacity pathway in mammalian cultured cells and is also conserved in Drosophila ${ }^{21,22}$. This pathway, termed CLIC/GEEC endocytosis, shares some features with the FEME pathway, as they both localize to the leading edge of migrating cells and involve tubular and ring-shaped pleomorphic carriers (ranging in diameter and length). However unlike FEME, which is stimulated by specific ligand-receptor interactions, CLIC/GEEC endocytosis is a constitutive pathway (that is, it continuously occurs in the cells that possess the pathway) that mediates the uptake of cargoes that are different from those using the FEME pathway ${ }^{16}$. Notably, this pathway is involved in the uptake of abundant surface proteins such as the hyaluronic acid receptor (CD44) and glycosylphosphatidylinositol-anchored proteins, and in some cells also mediates the uptake of substantial amounts of fluid and membrane. Using a real-time fluorescence assay and total internal reflection fluorescence microscopy to follow the early steps in this pathway, it was shown that there is minimal concentration of cargo proteins (glycosylphosphatidylinositol-anchored proteins) in the forming carriers before endocytosis ${ }^{23}$. This feature of the pathway has made its study difficult in comparison with FEME and CME, where clustering of cargo in the forming carrier makes for more straightforward observation by fluorescence microscopy. The CLICs mature into tubular GEECs. The pathway is regulated by ARF1/GBF1, the actin regulatory complex Arp2/3 and the small GTPase Cdc42, and is associated with a specific BAR domain protein, IRSp53, as well as GRAF1 (ref. ${ }^{23}$ ). In addition to this cytoplasmic machinery, a novel extracellular mechanism has been proposed for the uptake of specific CLIC/GEEC markers ${ }^{24}$. This process involves extracellular lectins called galectins that cluster glycosylated proteins and glycosphingolipids into nanoscale domains that then invaginate inwards to generate the tubular carrier. A similar mechanism is exploited by infectious agents such as the SV40 virus, cholera and Shiga toxins to enter the CLIC/GEEC endocytosis pathway (although note that these toxins are associated with a number 


\section{Box 2 | Summary of classical endocytic pathways}

Understanding the properties of each internalization pathway is crucial to understanding nanoparticle uptake. The table below provides a brief comparison of the parameters that should be considered for each internalization pathway. There is evidence to suggest that elements from these classical pathways can be involved in hybrid pathways; however, the behaviour of these hybrid pathways differs substantially from that of the classical pathways and should be considered separately.

\begin{tabular}{|c|c|c|c|c|c|c|}
\hline & CME & FEME & CLIC/GEEC & Caveolin & $\begin{array}{l}\text { Macrop } \\
\text { inocytosis }\end{array}$ & $\begin{array}{l}\text { Phago- } \\
\text { cytosis }\end{array}$ \\
\hline $\begin{array}{l}\text { Classical } \\
\text { size of } \\
\text { endocytic } \\
\text { vesicle } \\
\text { (diameter) }\end{array}$ & $\begin{array}{l}\sim 100 \mathrm{~nm} \\
\text { spherical }\end{array}$ & $\begin{array}{l}\sim 60-80 \mathrm{~nm} \\
\text { tubular } \\
\text { (may be } \\
\text { several } \\
\text { hundred } \\
\text { nm long) }\end{array}$ & $\sim 100 \mathrm{~nm}^{\mathrm{a}}$ & $\sim 60 \mathrm{~nm}$ & $>200 \mathrm{~nm}$ & $>200 \mathrm{~nm}^{\mathrm{b}}$ \\
\hline $\begin{array}{l}\text { Present in } \\
\text { all cells }\end{array}$ & Yes & Possiblyc & No & No & d & e \\
\hline $\begin{array}{l}\text { Validated } \\
\text { cargo }\end{array}$ & Transferrin & $\begin{array}{l}\text { Anti- } \beta_{1}{ }^{-} \\
\text {adrenergic } \\
\text { receptor }\end{array}$ & $\begin{array}{l}\text { Anti-CD44 } \\
\text { anti-CD98 }\end{array}$ & - & Dextran ${ }^{f}$ & $\begin{array}{l}\text { Chemically } \\
\text { killed } \\
\text { bacteria }\end{array}$ \\
\hline
\end{tabular}

asize is not well defined but is generally considered to be similar to that for CME and FEME.

${ }^{\mathrm{b}}$ Generally considered to involve particles larger than $500 \mathrm{~nm}$ in diameter but could play a role in uptake of smaller particles ${ }^{36}$.

'The FEME pathway has been found in most cell lines studied; however, only a limited number of cell lines have been explored.

${ }^{\mathrm{d}}$ Macropinocytosis is often upregulated in cultured cell lines and can be stimulated by growth factors.

eMany non-professional phagocytic cells appear to have the molecular machinery to undergo phagocytosis, but lack the classical receptors to bind cargo and initiate the process. Knockin studies of these receptors have shown that a number of classically non-phagocytic cells will phagocytose cargo if the appropriate receptor is expressed on their surfaces ${ }^{108}$.

fNote that if dextran is behaving as a true fluid phase marker it will be incorporated into all forming endocytic vesicles, particularly at low molecular masses $(<10,000 \mathrm{Da})$, and cannot only label the macropinocytic pathway. However, the magnitude of the pathway, and size of the forming macropinosomes, provide some apparent specificity for macropinosomes depending on the concentration and imaging conditions used, especially for very large dextrans (for example 2,000,000 Da).

of other endocytic pathways including FEME, CME and caveolae $)^{25}$. This mechanism is dependent on the multivalent nature of the galectin, which is secreted extracellularly in large quantities, and the coordinated interaction of the galectin with lipids and proteins, such as CD44 and integrins ${ }^{24}$. The pathway is particularly sensitive to changes in membrane tension and has been shown to be crucial for homoeostatic regulation of plasma membrane tension through the mechanotransducer protein vinculin ${ }^{26}$.

Macropinocytosis. Macropinosomes form by the actin-driven extension of plasma membrane sheets followed by enclosure of large volumes of the extracellular medium as the macropinosome folds back. The mechanisms involved in this process have been elegantly described in macrophages using lattice light sheet microscopy and have demonstrated the formation of tentpole-like actin extensions with sheets of membrane between ${ }^{27}$. Twisting of the 'tentpoles' causes the trapping of fluid at the base of the structure. After closure, the macropinosome undergoes a transformation as mem- brane tubules form and detach to recycle out membrane while the remaining vacuole shrinks. The macropinosome undergoes interactions with other compartments to acquire the characteristics of an early endosomal compartment.

The capacity for cells to macropinocytose material is highly dependent on the cell type and can be a constitutive or an induced process $^{28}$. Macrophages and dendritic cells exhibit high levels of constitutive macropinocytosis to sample the extracellular environment for pathogens ${ }^{29}$. Induction of macropinocytosis can occur through a range of stimuli, including receptor tyrosine kinase family receptors (for example EGFR), proteoglycans or G-protein-coupled receptors. Neutrophils have been shown to induce macropinocytosis in response to viral infections, and dendritic cells upregulate their native levels of macropinocytosis in response to treatment with lipopolysaccharide ${ }^{30}$.

In transformed cells with a RAS mutation, macropinocytosis plays a crucial role in uptake of protein to be used as a source of amino acids for fuel ${ }^{31}$. It has also been shown that breast and prostate cancer cells use macropinocytosis to increase trafficking of ErbB3 into the nucleus to promote proliferation ${ }^{32}$. Constitutive macropinocytosis levels vary in different cell lines in culture and undoubtedly also in different cells in vivo. Macropinocytosis can also be stimulated by growth factors in culture medium ${ }^{33}$. These factors make it important to ascertain the role of macropinocytosis in the target cells in vivo.

Phagocytosis. Phagocytosis involves the uptake of particulate material by professional phagocytes (polymorphonuclear neutrophils, monocytes and macrophages) and non-professional phagocytes ${ }^{34,35}$. This has been considered to involve the uptake of particles over $0.5 \mu \mathrm{m}$ in diameter, but the lower limits for phagocytic uptake are actually unclear and phagocytosis could be involved in the uptake of far smaller particles, including nanoparticles ${ }^{36}$. Phagocytosis is a crucial cellular process for uptake of cellular debris, including dead cells, and for the elimination of pathogenic microorganisms by cells of the innate immune system. Particles or microorganisms are engulfed by a tight-fitting membrane that extends around the particle until it is completely enclosed and scission from the plasma membrane occurs. The resultant intracellular vacuole, the early phagosome, matures into a degradative compartment containing a full complement of microbiocidal agents.

Phagocytosis is initiated by binding of particles to surface proteins such as scavenger receptors, which can recognize a diverse range of materials ${ }^{35}$, or by specific receptor interactions. The uptake of pathogens by macrophages is one of the best understood phagocytic processes. Clustering of surface receptors that recognize opsonized particles via their associated immunoglobulins activates Src family kinases. Sustained activation requires exclusion of key phosphatases, such as CD45 and CD148, from the site of phagocytosis. This process depends on the close apposition of the macrophage's enclosing phagosomal membrane and coordination with actin and integrin signalling networks. This signalling network strengthens the association of the phagosomal cup membrane with the phagocytosed particle, while the exclusion of CD45 and CD148 phosphatases accelerates the expansion of the phagocytic cup. Membrane extension and phagosome formation rely on the actin cytoskeleton, with actin filaments depolymerizing at the base of the cup and polymerizing at the tips of the lamellae as they extend over the particle. This relies on a series of small GTPases including Cdc42, Rac and RhoA working together with effectors such as WASP and Arp2/3 to generate a branched actin network. Actin remodelling also relies on transient changes in membrane lipids such as the consumption of $\operatorname{PtdIns}(4,5) \mathrm{P}_{2}$, through both hydrolysis and conversion to $\operatorname{PtdIns}(3,4,5) \mathrm{P}_{3}$. Phagocytic cup extension also requires dynamin-2, which regulates both actin dynamics and scission from the plasma membrane; however, the 
Table 1 | Summary of chemical endocytosis inhibitors

\begin{tabular}{|c|c|c|c|c|c|c|c|c|c|}
\hline \multirow[t]{2}{*}{ Inhibitor } & \multirow[t]{2}{*}{ Target pathway } & \multirow[t]{2}{*}{ Mode of action } & \multicolumn{6}{|c|}{ Pathways affected } & \multirow[t]{2}{*}{ Comments } \\
\hline & & & CME & FEME & Cav & CLIC & Macro & Phago & \\
\hline $\begin{array}{l}\text { 2-deoxy-D-glucose/ } \\
\text { sodium azide }\end{array}$ & $\begin{array}{l}\text { All energy- } \\
\text { dependent } \\
\text { pathways }\end{array}$ & $\begin{array}{l}\text { Decreases ATP and } \\
\text { NADH by inhibiting } \\
\text { glycolysis }\end{array}$ & $\checkmark$ & $\checkmark$ & $\checkmark$ & $\checkmark$ & $\checkmark$ & $\checkmark$ & \\
\hline Hypertonic sucrose ${ }^{79}$ & CME & $\begin{array}{l}\text { Prevents disassembly of } \\
\text { clathrin cage }\end{array}$ & $\checkmark$ & • & & & • & & Non-specific ${ }^{80}$ \\
\hline Chlorpromazine & CME & $\begin{array}{l}\text { Mechanism unknown }{ }^{82} \text {, } \\
\text { but probably inhibits } \\
\text { AP2 }^{83}\end{array}$ & $\checkmark$ & $\bullet$ & $\bullet$ & $\bullet$ & & & $\begin{array}{l}\text { Increases the uptake of } \\
\text { lactosylceramide, which } \\
\text { is not internalized by } \\
\mathrm{CME}_{i}^{; 4} \text { inhibits FEME }\end{array}$ \\
\hline Chloroquine $^{83}$ & CME & $\begin{array}{l}\text { Affects the function } \\
\text { of clathrin and } \\
\text { clathrin-coated vesicles }{ }^{85}\end{array}$ & $\checkmark$ & & & & & & $\begin{array}{l}\text { Also inhibits endosome } \\
\text { acidification }\end{array}$ \\
\hline Dynasore $^{89} \&$ Dyngo ${ }^{90}$ & $\begin{array}{l}\text { All dynamin- } \\
\text { dependent } \\
\text { pathways } \\
(\mathrm{CME} \& \mathrm{FEME})\end{array}$ & $\begin{array}{l}\text { Block the GTPase activity } \\
\text { of dynamin }\end{array}$ & $\checkmark$ & $\checkmark$ & & & $\bullet$ & $\bullet$ & $\begin{array}{l}\text { Can also interfere with } \\
\text { actin, as shown in } \\
\text { triple-knockout } \\
\text { dynamin cells }\end{array}$ \\
\hline Methyl- $\beta$-cyclodextrin ${ }^{92}$ & $\begin{array}{l}\text { Lipid rafts/ } \\
\text { cholesterol-enriched } \\
\text { microdomains/ } \\
\text { caveolae }\end{array}$ & $\begin{array}{l}\text { Removes cholesterol } \\
\text { from the plasma } \\
\text { membrane }\end{array}$ & ) & $\bullet$ & $\checkmark$ & $\checkmark$ & $\bullet$ & $\bullet$ & $\begin{array}{l}\text { Interferes with other } \\
\text { uptake mechanisms } \\
\text { because of changes in } \\
\text { membrane fluidity }{ }^{93}\end{array}$ \\
\hline Nystatin & $\begin{array}{l}\text { Lipid rafts/ } \\
\text { cholesterol-enriched } \\
\text { microdomains/ } \\
\text { caveolae }\end{array}$ & Binds to cholesterol ${ }^{94}$ & & $\bullet$ & $\checkmark$ & $\checkmark$ & $\bullet$ & $\bullet$ & $\begin{array}{l}\text { Interferes with other } \\
\text { uptake mechanisms } \\
\text { because of changes in } \\
\text { membrane fluidity }{ }^{93}\end{array}$ \\
\hline Cytochalasin D & $\begin{array}{l}\text { Macropinocytosis } \\
\text { and phagocytosis }\end{array}$ & Depolymerizes F-actin ${ }^{98}$ & $\bullet$ & $\bullet$ & $\bullet$ & $\bullet$ & $\checkmark$ & $\checkmark$ & $\begin{array}{l}\text { Interference with actin } \\
\text { polymerization affects } \\
\text { multiple pathways }\end{array}$ \\
\hline Amiloride (EIPA) ${ }^{100}$ & Macropinocytosis & $\begin{array}{l}\text { Inhibits } \mathrm{Na}^{+} \text {channels and } \\
\mathrm{Na}^{+} / \mathrm{H}^{+} \text {exchange }\end{array}$ & \% & $\bullet$ & & & $\checkmark$ & & $\begin{array}{l}\text { Reports have shown } \\
\text { inhibition }{ }^{100} \text {, or lack of } \\
\text { inhibition }{ }^{102} \text {, of EGFR } \\
\text { uptake; has been shown } \\
\text { to inhibit FEME }{ }^{16}\end{array}$ \\
\hline
\end{tabular}

$\checkmark$ Proposed target pathway. $\bullet$ Other, 'off-target', pathways. Requires specific conditions to prevent 'off-target' effects.

mechanism of action is probably different from other internalization mechanisms $s^{37}$.

Once the phagosome forms, a series of complex maturation events occurs as the phagosome fuses with endosomal compartments and acquires the characteristics of a late-endosomal/lysosomal compartment. These maturation steps involve a series of complex membrane trafficking steps, including sequential acquisition of Rab GTPases, microtubule-dependent trafficking through dynein/dynactin, recruitment of elements of the autophagosomal machinery and selective retrieval of membrane-associated components from the forming phagolysosomes ${ }^{38}$.
Caveolae: limited evidence for nanoparticle uptake. An extensive literature links caveolae to endocytosis. This includes decades of work in vascular endothelia, in which caveolae have been proposed to mediate transcellular transport from the lumen of vessels across the endothelium and into tissues. In non-endothelial cells, caveolae have also been implicated in a number of endocytic processes including uptake of toxins, viruses, whole bacteria, lipids and a range of nanoparticles. Each of these pathways remains somewhat controversial, particularly as studies using genetic knockout of caveolar components have not generally shown a dependence on caveolae for endocytic uptake in any system ${ }^{12,39}$. For example, 
mice lacking caveolae show efficient transport of albumin from the bloodstream to tissues ${ }^{40}$, while viruses and toxins shown to associate with caveolae are not dependent on caveolae for their infectious entry and toxicity ${ }^{41}$. A set of criteria that can be used to assess uptake via caveolae has been discussed elsewhere ${ }^{42}$.

Caveolae are characterized by a unique morphology as seen by electron microscopy: a bulb-shaped pit of approximately $60 \mathrm{~nm}$ diameter connected to the plasma membrane by a slightly smaller neck ${ }^{43}$. Caveolae are very abundant in some cell types but absent from others (for example neurons and many blood cells). Many commonly used mammalian cell lines also lack caveolae, including PC3 cells and at least some MCF7 strains (Box 3). This cell- and tissue-specific distribution is very different from clathrin-coated pits, which show a fairly constant density in different cell types. Caveolae are generated by caveolins and cavins working together with accessory proteins such as Eps15 homology domain-containing 2 (EHD2), pacsin/syndapins and ROR1 $\left(\right.$ ref. ${ }^{12}$ ). Cavins are peripheral membrane proteins that associate primarily with caveolae under steady-state conditions. However, caveolins are integral membrane proteins that associate with a number of cellular compartments, including caveolae, endosomes, the Golgi complex and lipid droplets. This wide distribution of caveolins has led in part to confusion over the role of caveolins in endocytic pathways. Loss of caveolae occurs when the expression of caveolin-1 (CAV1; in non-muscle cells), caveolin-3 (CAV3; striated muscle cells) or cavin1 (in both muscle and non-muscle cells) is ablated. This provides a way to test whether uptake of specific markers is dependent on caveolae.

The role of caveolae in endocytic processes, as endocytic carriers analogous to clathrin-coated pits, is not clear despite the dogma that has developed in some research fields. A number of factors have added confusion to the field. First, a number of studies have relied on inhibitors to test for the involvement of caveolae. Invariably these inhibitors have insufficient specificity to prove the involvement of caveolae (Table 1). For example, cholesterol perturbation not only disrupts caveolae but also perturbs CLIC/GEEC endocytosis and FEME, even under conditions where transferrin uptake via clathrin-coated pits is unaffected ${ }^{14,16,41,44}$. Second, colocalization with caveolin has been considered an indicator of caveolar endocytosis. In fact, overexpression of caveolin-1 leads to its association with a number of endocytic pathways and compartments, as the levels of endogenous cavin limit caveolar formation. An example of this is the proposed association of caveolin-1 with a novel compartment called the caveosome $e^{45}$, subsequently proposed by the original authors to be an artefact of caveolin-1 overexpression ${ }^{46}$. Experiments using fluorescently tagged caveolin-1 must also be performed with care in view of studies proposing that both aminoand carboxy-terminal-tagged proteins can act as dominant-negative inhibitors ${ }^{45}$.

Another important aspect of studies proposing involvement of caveolae is whether the particle or agent of interest can be physically accommodated within a caveola. The interior of caveolae, around $50 \mathrm{~nm}$ in diameter, can only accommodate relatively small cargo. Large cargo, such as bacteria ${ }^{47}$ and larger nanoparticles ${ }^{48}$, have been proposed to use caveolae, but it is not clear how the caveola structure could accommodate materials greater than the diameter of this pathway. The structure of vesicles to accommodate these particles would need to be dramatically modified and so they should not be considered caveolae. However, caveolar proteins could still be hijacked to facilitate the internalization process. We suggest that if such mechanisms are proven they should be very clearly differentiated from bona fide caveolar endocytosis, which should be confined to budding of intact caveolae with cargo.

In conclusion, without further evidence, it is difficult to establish whether any nanoparticles require caveolae for endocytosis, at least in non-endothelial cells. Use of genetically modified cells and organisms should be able to resolve many of these issues in the future (Table 2).

Nanoparticle uptake: general considerations. Many notable studies investigating nanoparticle size, shape, charge and targeting were performed before we fully understood the roles of the CLIC/GEEC and FEME pathways. Furthermore, recent advances in our understanding of how pharmacological inhibitors interact with multiple endocytosis pathways also calls for us to reassess the properties that govern endocytosis $^{49}$. The pathways of CME, FEME, CLIC/GEEC and caveolae are all associated with carriers with a diameter smaller than $200 \mathrm{~nm}$, which means it is unlikely that particles larger than $200 \mathrm{~nm}$ can be internalized by these routes. Particles larger than $200 \mathrm{~nm}$ will typically be internalized by macropinocytosis or phagocytosis (but note that proteins such as clathrin and dynamin can still play a crucial role in these pathways $)^{50,51}$. Reports that particles larger than $200 \mathrm{~nm}$ are endocytosed by caveolae conflict with our current understanding of the caveola structure ${ }^{48}$. Below $100 \mathrm{~nm}$, the size of the nanoparticle plays a less important role in possible routes of uptake, as the geometry of each pathway can readily accommodate small nanoparticles.

When nanoparticles are placed in cell media or are injected in vivo, serum proteins quickly adsorb to the surface of the nanoparticle, forming a protein corona. These adsorbed proteins, such as vitronectin, can induce binding to specific cell surface receptors, which in turn can influence the uptake pathway ${ }^{52}$. The formation of a protein corona can also cause particles to aggregate before they reach the cell, causing the effective nanoparticle size to be significantly larger than the size measured ex vivo in water or PBS. We have recently helped to draft the MIRIBEL standard for reporting the properties of nanoparticles, and feel that reporting the full characterization of these materials will help with the comparison of different studies ${ }^{53}$.

\section{Tools to study mechanisms of uptake}

A considerable research effort has been, and is still being, expended on understanding the pathways by which nanoparticles are internalized $^{9}$. Many of these studies employ inhibitors, and particularly in the nanoparticle literature many rely solely on the use of inhibitors and cell culture models. A pertinent question is whether these studies have helped the field to progress, and in particular whether their use helps in the design and application of new therapeutics (here we are not discussing the use of endocytosis inhibitors themselves as therapeutic agents, something that has considerable potential) ${ }^{6}$.

Aside from the question of the importance of dissecting the pathways of endocytosis of nanoparticles in cultured cells, a crucial question is how specific these inhibitors are. Many inhibitors considered specific for macropinocytosis also inhibit FEME (Table 1). In fact, in one study all tested inhibitors of macropinocytosis and clathrin-independent endocytosis also inhibited FEME ${ }^{16}$. A number of inhibitors of clathrin-coated pit endocytosis also inhibit FEME, including chlorpromazine and potassium depletion, whereas cholesterol depletion to inhibit uptake via caveolae also inhibits the CLIC/GEEC and FEME pathways.

The non-specific and cross-reactive nature of these inhibitors means that it is important to confirm the activity of the inhibitors ${ }^{54}$. This can be achieved using markers that have been extensively validated to be specifically internalized by particular pathways (Box 1). Use of these markers not only confirms the activity of the inhibitor but can also give information about non-specific inhibition.

The methods used to study endocytosis are also important. The uptake of nanoparticles into cells is typically investigated by fluorescently labelling materials and measuring uptake using fluorescence microscopy or flow cytometry. One challenge is accurately distinguishing material bound to the plasma membrane from internalized material ${ }^{55}$. Confocal microscopy offers a low-throughput 
Box 3 | Summary of endocytic mechanisms in common human cell lines

Understanding the biology of the cells being studied and the internalization pathways they possess is crucial to understanding nanoparticle uptake. The table below provides a brief summary of the endocytic mechanisms present in seven commonly studied human cell lines. While all cell lines possess CME machinery, not all uptake mechanisms are ubiquitous. Of note, PC3 cells (lack cavins) ${ }^{105}$, HEK293 cells (lack cavin1), Caco 2 cells (lack CAV1) and many MCF7 cell lines (lack caveolins and cavins) ${ }^{109}$ do not possess caveolae. Despite this, a number of studies have concluded that nanoparticles are internalized via a caveola-mediated process. Furthermore, HeLa cells do not possess the CLIC/GEEC pathway, as judged by a number of criteria ${ }^{26,110}$; therefore, it cannot be assumed that this pathway is present in all cells. Phagocytosis is initiated by binding to surface receptors that are not present on all cells. However, phagocytosis can be induced in some non-phagocytic cells if they are modified to express these receptors ${ }^{111}$. Recent work has also suggested that dividing cells in culture differ from the same cells in a non-dividing quiescent state in their endocytic uptake pathways $^{109}$. All of these examples illustrate the importance of understanding the limitation of cell culture models and the need to develop assays for in vivo studies of endocytosis.

\begin{tabular}{|c|c|c|c|c|c|}
\hline \multirow[t]{2}{*}{ Cell line } & \multicolumn{5}{|c|}{ Internalization pathway } \\
\hline & $\mathrm{CME}^{112}$ & FEME & Cav & CLIC/GEEC & Macro"1 \\
\hline A549 & $\checkmark$ & $S^{a 114}$ & b115,116 & $\boldsymbol{J}^{\text {a117 }}$ & $\boldsymbol{S}^{\mathrm{c} 118}$ \\
\hline MCF7 & $\checkmark$ & ? & $X^{109}$ & $?$ & $\checkmark$ \\
\hline PC3 & $\checkmark$ & $?$ & $\times^{105}$ & ? & $\checkmark$ \\
\hline HeLa & $\checkmark$ & $\sqrt{ }^{119}$ & $\boldsymbol{S}^{120}$ & $Q^{26,110}$ & $\checkmark$ \\
\hline HepG2 & $\checkmark$ & ? & \% & $\boldsymbol{J}^{121}$ & $\checkmark$ \\
\hline HEK293 & $\checkmark$ & $\boldsymbol{J}^{\mathrm{a} 122}$ & $x^{123}$ & i $^{124}$ & $\checkmark$ \\
\hline Caco2 & $\checkmark$ & $\boldsymbol{J}^{125}$ & $X^{126}$ & $\boldsymbol{J}^{127}$ & $\checkmark$ \\
\hline
\end{tabular}

$\checkmark$ Present.

$\checkmark \checkmark$ Upregulated.

$\times$ Not present due to the lack of key molecular machinery.

$Q$ Not present on the basis of comparative cellular studies. This does not rule out modification of the classical pathway.

? Not investigated.

Conflicting evidence.

andirect evidence.

bonflicting evidence but probably very low.

${ }^{c}$ A549 cells have the KRAS mutation, which has been shown to upregulate macropinocytosis $\mathrm{s}^{31,32}$.

Note that the table is only an indication of the variability that can occur in commonly used lines. For many cultured cells definitive evidence is lacking, and we have noted considerable variation in published findings even with apparently identical cell lines. It is also often difficult to demonstrate the lack of a particular pathway, such as CLIC/GEEC, rather than the existence of a modified version of this pathway in a specific cell type. However, the table serves to illustrate the dangers of assuming that all pathways exist in all cultured mammalian cell types, also illustrated by comparison of caveolae for which structural components and well defined ultrastructural morphology are available.

technique to qualitatively determine if nanoparticles are inside the cell; however, it can be difficult to distinguish between material that is internalized, but close to the cell surface, and material bound to the outer plasma membrane. Flow cytometry is a high-throughput method for rapidly quantifying the association of material with cells; however, by default flow cytometry quantifies total association, rather than distinguishing internalized material from surface-bound material. Recently a number of new techniques have been developed that allow rapid quantification of uptake using flow cytometry ${ }^{56,57}$. The specific techniques to quantify uptake are beyond the scope of this Review; however, we have recently reviewed the latest advances in quantifying cellular uptake elsewhere ${ }^{58}$. A common technique to synchronize internalization and normalize nanoparticle binding is to initially incubate nanoparticles with cells at $4^{\circ} \mathrm{C}$ to inhibit uptake. Unbound particles are then washed away and the cells returned to $37^{\circ} \mathrm{C}$ to initiate uptake. The synchronized internalization allows for easy interpretation of internalization kinetics. However, CLIC/GEEC endocytosis has been reported to recover slowly after a low-temperature incubation while clathrin-dependent endocytosis recovers rapidly ${ }^{10}$. This has the potential to underrepresent the importance of CLIC/GEEC pathway and overestimate the importance of CME if a low-temperature protocol is used. To visualize the FEME pathway it is important that the cells are fixed at $37^{\circ} \mathrm{C}$, which suggests that temperature may also influence this pathway ${ }^{16}$. Furthermore, a number of endocytosis inhibitors require serum-free media; however, it has been established that serum stimulates FEME but not CLIC/GEEC endocytosis, while serum starvation downregulates FEME ${ }^{16,21}$. Consequently, decoupling the role of FEME from other pathways using small-molecule inhibitors can be challenging. Increasingly researchers are employing incubation at $37^{\circ} \mathrm{C}$ with no cooling step and a serum-containing medium to minimize these problems.

Knockout of key components in the internalization pathways, or expression of dominant-negative inhibitors, if performed carefully with appropriate controls, can overcome these problems (Table 2; although note that these inhibitors can also have off-target effects because some components can be shared by different pathways or can influence these pathways ${ }^{13}$. These include the expression of the BAR domain of endophilin lacking its N-terminal amphipathic helix (b) for the FEME pathway, caveolin-1 or cavin1 knockout for the caveolar pathway, and the use of dynamin-knockout cells in which all three isoforms are genetically ablated or can be conditionally downregulated. RNA interference is a valuable tool to achieve knockdown of these key proteins and has been used to interrogate the role of different endocytic pathways in the endocytosis of nanomaterials ${ }^{8}$. siRNA can also be used to screen numerous proteins either individually or in concert to assess their implications in different endocytic pathways ${ }^{59,60}$. Although this approach is likely to be more specific than pharmacological inhibitors, the highly intricate nature of these endocytic pathways, which may share protein components (an aspect that may not be well understood currently), means that validation is critical to avoid affecting multiple pathways. siRNA-mediated knockdown can also suffer from off-target effects.

\section{Fate}

For delivery of therapeutic nanoparticles, internalization is only the first step. The subsequent trafficking substantially impacts the efficacy of the delivery system. For delivery of the therapeutic to its site of action, typically in the cytosol or nucleus, a number of questions arise. First, does the endocytic pathway matter? Second, can we specifically exploit the distinct characteristics of specific pathways to benefit drug or nanoparticle uptake and trafficking? Third, what do we know about these pathways in vivo? Surprisingly, in view of the importance of the question and the number of research papers devoted to nanoparticle or drug delivery, the answer to this last question is that very little is known. These questions will be addressed in turn.

There is strong evidence to suggest that all major endocytosis pathways are capable of delivering their contents to the early endosome through a Rab5/EEA1-dependent trafficking pathway ${ }^{61}$. 
Table 2 | Summary of genetic inhibitors of endocytosis

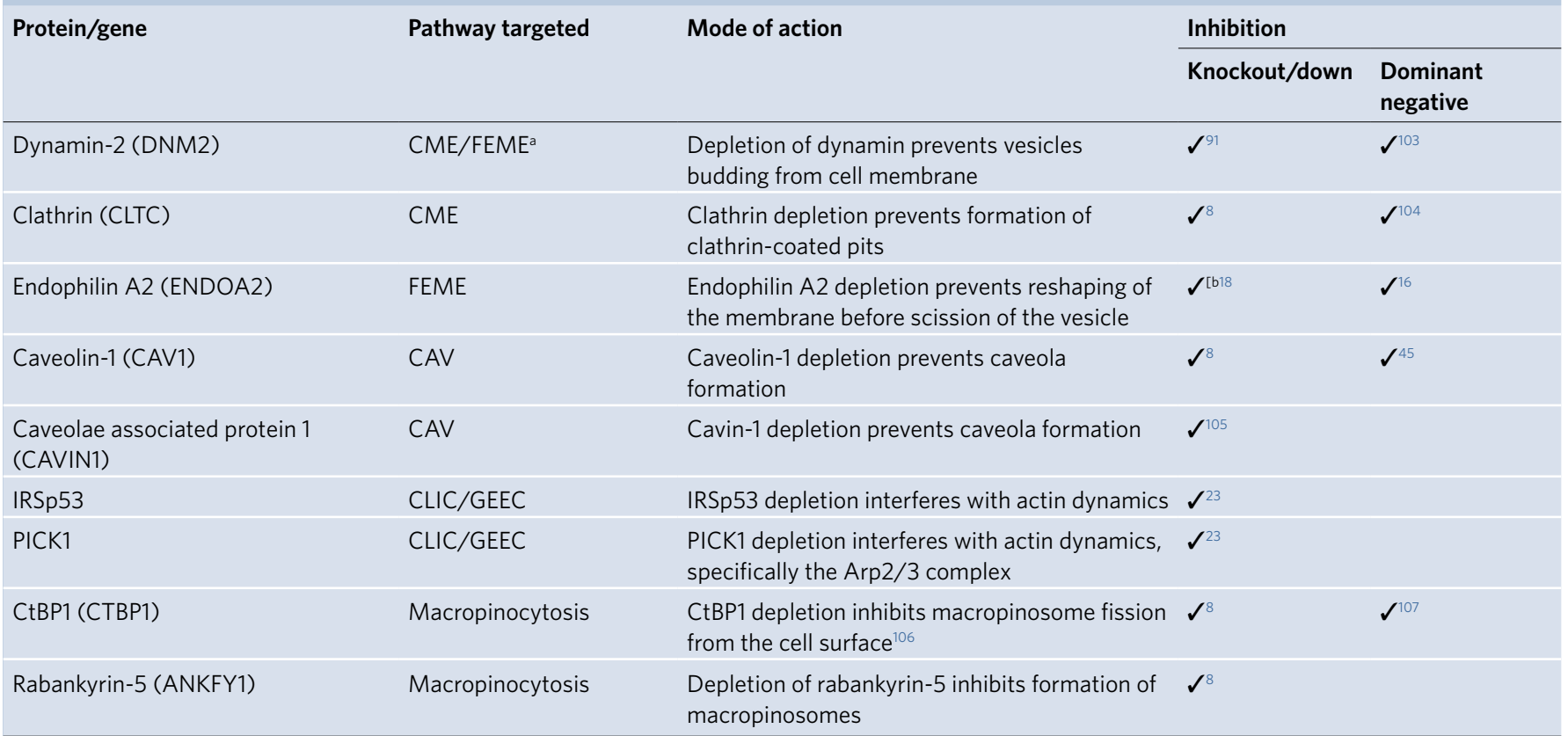

${ }^{a}$ Dynamin-2 is implicated in phagocytosis; however, only specialized cells are phagocytic. ${ }^{\circ}$ For unambiguous knockdown it may be necessary to knock down all three forms of endophilin, although some show inhibition by only knocking down endophilin A2.

From the early endosome, cargo can be recycled back to the cell surface either directly or via the Rab11-positive recycling endosome, or can remain with the vacuolar portion of the early endosome as it converts to a late-endosomal compartment and is eventually trafficked to the lysosomes. The majority of studies investigating the fate of nanoparticles and their cargo show substantial accumulation in the lysosomes.

In addition to the classical pathway of uptake to the EEA1-positive early endosome, internalized cargo in clathrin-coated vesicles is also delivered to the APPL1-positive early endosome, a parallel stable sorting compartment ${ }^{62,63}$. This compartment may be not an essential station for bulk endocytic cargo but rather a signalling compartment $^{62}$. Traffic to the APPL endosome may delay transport into the EAA1 endosome, where recycling or degradation could occur to allow prolonged signalling.

It is important to note that cargo endocytosed by the same mechanism can be sorted into different endosomal compartments, and that cargo endocytosed by different mechanisms can be sorted into the same endosomal compartment. CD44, CD98, CD147 and MHCI enter cells via a clathrin-independent pathway, and colocalize to the same endocytic vesicles after $5 \mathrm{~min}$ (ref. ${ }^{64}$ ). However, CD44, CD98 and CD147 are rapidly sorted in a different way from MHCI into EEA1-negative endosomal compartments and avoid the degradative lysosomal pathway. This altered trafficking is controlled by sequences in the cytoplasmic domain of the receptors ${ }^{65}$. It has yet to be tested whether this distinct trafficking route can influence the fate of nanoparticles.

The fate of nanoparticles can be driven by a number of factors that relate to the mechanism of internalization. These include the rate of internalization, the percentage of material internalized and signalling on the cytosolic side of the internalized receptor. There is some evidence to suggest that certain internalization pathways can influence subsequent cellular trafficking; however, it is not clear if it is the internalization pathway or signalling from the receptor that is the major driver of this trafficking. As an example of differential trafficking, liposomes with high surface densities of octa-arginine have been shown to increase macropinocytosis, and had higher transfection efficiencies than lower-density octa-arginine liposomes, which were internalized by other pathways ${ }^{66}$.

The delivery requirements of small-molecule drugs and large macromolecules such as proteins or RNA/DNA are quite different. Small-molecule drugs are generally synthesized such that their properties allow the passive diffusion across cell membranes. Therefore, simply achieving accumulation in a target tissue without regard for the subsequent internalization mechanism may be sufficient to attain enhanced drug delivery to a specific subset of cells. Comparatively large macromolecules are unable to passively diffuse across these membranes due to their size and polarity, and therefore rely on endosomal escape to reach sites of action within the cytosol or nucleus ${ }^{67}$. It remains to be seen whether endosomal escape is dependent on the trafficking route within which the internalized material finds itself. If certain trafficking vesicles result in increased endosomal escape (perhaps due to specific lipid content) ${ }^{68}$, it may be possible to target specific endocytic compartments to control the therapeutics' fate and enhance cytosolic delivery. Methods to understand endosomal escape and engineer nanoparticles to promote cytosolic delivery have been reviewed recently ${ }^{67,69,70}$, and are beyond the scope of this Review.

\section{Endocytic pathways in vivo}

We have highlighted the variation in endocytic pathways in different commonly used laboratory cell lines. It is clear that there will be even more variation in endocytosis between different cell types in vivo, reflecting the particular properties of these cells, their physiological functions and the ever changing local environment of cells in different tissues within a whole organism. The organization of the endosomal circuits differs between cell types in vivo ${ }^{71}$, and particular cell types, such as cells of the kidney proximal tubules, have evolved high-capacity internalization mechanisms. Even cells of the same type grown in culture under different conditions can dramatically remodel their endocytic pathways as they change from a dividing to a quiescent state ${ }^{72}$, and so it is not surprising that the few studies that have compared endocytosis by cells in culture with their in vivo counterparts have shown notable differences in 
endocytic pathways ${ }^{73}$. Very little is still known of the magnitude of different endocytic pathways in physiological cell types in different tissues and how these differences impact on the delivery of nanoparticles into the cells of interest in vivo. However, the application of intravital subcellular microscopy, including the use of light microscopy techniques such as spinning-disc, confocal and multiphoton microscopy in live animals, is now starting to provide new insights into the uptake of biological and therapeutic agents in vivo ${ }^{74-76}$. Another important aspect of delivery in vivo, which lies outside the scope of this Review, is transport from the bloodstream across the endothelium to the target cells, and the reader is referred to a recent review on this subject ${ }^{77}$. A crucial future aim must be to develop tractable systems that go beyond the culture dish and into more physiological systems.

\section{Conclusion}

Nanoparticle delivery systems have the potential to improve the treatment of various diseases. Understanding how these nanoparticles are internalized by cells and then processed within the cells is critical for understanding how nanoparticles can reach their site of action. Here we present an overview of the current understanding of endocytosis, and the limitations of current experimental techniques, in the hope of stimulating research directed at understanding how a nanoparticle is internalized in target cells in vivo, and how an understanding of this process can dictate therapeutic strategies. Increased understanding of the process can potentially help harness cell biological mechanisms for more efficient delivery. Whether we can actually modulate the endocytic process to favour therapeutic delivery via a productive pathway is still unclear, although modulation of endocytosis in the clinic is now becoming a real possibility. Simple analysis of nanoparticle uptake mechanisms through the use of one or two non-specific pharmaceutical inhibitors adds little knowledge to our understanding of nanoparticle-cell interactions. It is important to recognize that most endocytosis inhibitors are working on multiple pathways (Table 1), which makes it difficult to draw definitive conclusions about the endocytic pathways. Instead, genetic knockouts or dominant-negative proteins may provide a more specific approach to understand uptake. There are a number of conflicting reports in the literature that conclude specific endocytic pathways without using the most up-to-date tools and knowledge. Compounding this, a number of commonly reported pathways are not ubiquitous to all cells.

Aspects of nanoparticle design, such as size, shape and surface chemistry, are thought to influence the route of internalization. However, many of these studies were performed before we developed our current understanding of endocytosis mechanisms, and it is important to re-evaluate them with the most up-to-date understanding.

While cell culture systems are vital for elucidating the fundamental mechanisms, molecules and pathways of endocytosis, it is important to understand their limitations. In vitro studies help answer specific questions about the pathways being studied, but they do not answer the wider question of whether these mechanisms are relevant in vivo. We see use of systems that more faithfully reflect the in vivo environment as vital. Ultimately this might require the use of whole-animal systems and development of new tools to study these in vivo. This does not mean that cell culture studies are not valid; rather, it means that in vitro studies should be informed by in vivo evidence. The use of intermediate systems such as explants or organoids, in which endocytosis can be manipulated in vitro, might be an excellent intermediate step in characterization. The study of EGFR endocytosis in live ex vivo human tumour biopsies represents an interesting step in this direction ${ }^{6,78}$. Creating nanoparticle systems with this in mind will aid the development and testing of delivery systems in a rational scientific fashion and most importantly should improve the efficacy of nanoparticles in therapeutic applications.

Received: 25 May 2020; Accepted: 19 January 2021; Published online: 12 March 2021

\section{References}

1. Karasneh, G. A. \& Shukla, D. Herpes simplex virus infects most cell types in vitro: clues to its success. Virol. J. 8, 481 (2011).

2. Tang, R. et al. Direct delivery of functional proteins and enzymes to the cytosol using nanoparticle-stabilized nanocapsules. ACS Nano 7, 6667-6673 (2013).

3. Mout, R. et al. General strategy for direct cytosolic protein delivery via protein-nanoparticle co-engineering. ACS Nano 11, 6416-6421 (2017).

4. Wilhelm, S. et al. Analysis of nanoparticle delivery to tumours. Nat. Rev. Mater. 1, 16014 (2016)

5. Tsoi, K. M. et al. Mechanism of hard-nanomaterial clearance by the liver. Nat. Mater. 15, 1212-1221 (2016).

6. Chew, H. Y. et al. Endocytosis inhibition in humans to improve responses to ADCC-mediating antibodies. Cell 180, 895-914.e27 (2020).

7. Yamashita, T., Takahashi, Y. \& Takakura, Y. Possibility of exosome-based therapeutics and challenges in production of exosomes eligible for therapeutic application. Biol. Pharm. Bull. 41, 835-842 (2018).

8. Gilleron, J. et al. Image-based analysis of lipid nanoparticle-mediated siRNA delivery, intracellular trafficking and endosomal escape. Nat. Biotechnol. 31, 638-646 (2013).

Detailed ultrastructural analysis of lipid nanoparticle uptake, and siRNA delivery, in cultured cells and in mouse liver.

9. Sahay, G., Alakhova, D. Y. \& Kabanov, A. V. Endocytosis of nanomedicines. J. Control. Release 145, 182-195 (2010).

10. Johannes, L., Parton, R. G., Bassereau, P. \& Mayor, S. Building endocytic pits without clathrin. Nat. Rev. Mol. Cell Biol. 16, 311-321 (2015).

11. Thottacherry, J. J., Sathe, M., Prabhakara, C. \& Mayor, S. Spoiled for choice: diverse endocytic pathways function at the cell surface. Annu. Rev. Cell Dev. Biol. 35, 55-84 (2019).

A comprehensive topical review of clathrin-dependent and clathrin-independent endocytic pathways.

12. Parton, R. G. Caveolae: structure, function, and relationship to disease. Annu. Rev. Cell Dev. Biol. 34, 111-136 (2018).

13. Kumari, S., MG, S. \& Mayor, S. Endocytosis unplugged: multiple ways to enter the cell. Cell Res. 20, 256-275 (2010).

14. Chaudhary, N. et al. Endocytic crosstalk: cavins, caveolins, and caveolae regulate clathrin-independent endocytosis. PLoS Biol. 12, e1001832 (2014).

15. Damke, H., Baba, T., van der Bliek, A. M. \& Schmid, S. L. Clathrin-independent pinocytosis is induced in cells overexpressing a temperature-sensitive mutant of dynamin. J. Cell Biol. 131, 69-80 (1995).

16. Boucrot, E. et al. Endophilin marks and controls a clathrin-independent endocytic pathway. Nature 517, 460-465 (2015). Characterization of a novel endophilin-dependent pathway, termed FEME.

17. Brown, C. M. \& Petersen, N. O. Free clathrin triskelions are required for the stability of clathrin-associated adaptor protein (AP-2) coated pit nucleation sites. Biochem. Cell Biol. 77, 439-448 (1999).

18. Ehrlich, M. et al. Endocytosis by random initiation and stabilization of clathrin-coated pits. Cell 118, 591-605 (2004).

19. Veiga, E. et al. Invasive and adherent bacterial pathogens co-opt host clathrin for infection. Cell Host Microbe 2, 340-351 (2007).

20. Li, Z. et al. Shape effect of glyco-nanoparticles on macrophage cellular uptake and immune response. ACS Macro Lett. 5, 1059-1064 (2016).

21. Howes, M. T. et al. Clathrin-independent carriers form a high capacity endocytic sorting system at the leading edge of migrating cells. J. Cell Biol. 190, 675-691 (2010)

22. Hemalatha, A., Prabhakara, C. \& Mayor, S. Endocytosis of Wingless via a dynamin-independent pathway is necessary for signaling in Drosophila wing discs. Proc. Natl Acad. Sci. USA 113, E6993-E7002 (2016).

23. Sathe, M. et al. Small GTPases and BAR domain proteins regulate branched actin polymerisation for clathrin and dynamin-independent endocytosis. Nat. Commun. 9, 1835 (2018).

24. Lakshminarayan, R. et al. Galectin-3 drives glycosphingolipid-dependent biogenesis of clathrin-independent carriers. Nat. Cell Biol. 16, 592-603 (2014).

25. Sandvig, K. \& van Deurs, B. Endocytosis, intracellular transport, and cytotoxic action of Shiga toxin and ricin. Physiol. Rev. 76, 949-966 (1996).

26. Thottacherry, J. J. et al. Mechanochemical feedback control of dynamin independent endocytosis modulates membrane tension in adherent cells. Nat. Commun. 9, 4217 (2018).

27. Condon, N. D. et al. Macropinosome formation by tent pole ruffling in macrophages. J. Cell Biol. 217, 3873-3885 (2018). 
28. Lin, X. P., Mintern, J. D. \& Gleeson, P. A. Macropinocytosis in different cell types: similarities and differences. Membranes 10, 177 (2020).

29. Kerr, M. C. \& Teasdale, R. D. Defining macropinocytosis. Traffic 10, 364-371 (2009).

30. Lim, J. P. \& Gleeson, P. A. Macropinocytosis: an endocytic pathway for internalising large gulps. Immunol. Cell Biol. 89, 836-843 (2011).

31. Commisso, C. et al. Macropinocytosis of protein is an amino acid supply route in Ras-transformed cells. Nature 497, 633-637 (2013).

Macropinocytosis is shown to have a crucial role in providing nutrients for cancer cells through the internalization and catabolism of extracellular proteins.

32. Ha, K. D., Bidlingmaier, S. M. \& Liu, B. Macropinocytosis exploitation by cancers and cancer therapeutics. Front. Physiol. 7, 381 (2016).

33. Palm, W. Metabolic functions of macropinocytosis. Philos. Trans. R. Soc. B 374, 20180285 (2019).

34. Niedergang, F. \& Grinstein, S. How to build a phagosome: new concepts for an old process. Curr. Opin. Cell Biol. 50, 57-63 (2018).

35. Lim, J. J., Grinstein, S. \& Roth, Z. Diversity and versatility of phagocytosis: roles in innate immunity, tissue remodeling, and homeostasis. Front. Cell. Infect. Microbiol. 7, 191 (2017).

36. Desjardins, M. \& Griffiths, G. Phagocytosis: latex leads the way. Curr. Opin. Cell Biol. 15, 498-503 (2003).

37. Doherty, G. J. \& McMahon, H. T. Mechanisms of endocytosis. Annu. Rev. Biochem. 78, 857-902 (2009).

38. Harrison, R. E., Bucci, C., Vieira, O. V., Schroer, T. A. \& Grinstein, S. Phagosomes fuse with late endosomes and/or lysosomes by extension of membrane protrusions along microtubules: role of Rab7 and RILP. Mol. Cell. Biol. 23, 6494-6506 (2003).

39. Parton, R. G. et al. Caveolae: the FAQs. Traffic 21, 181-185 (2020).

40. Schubert, W. et al. Microvascular hyperpermeability in caveolin-1 (-/-) knock-out mice. J. Biol. Chem. 277, 40091-40098 (2002).

41. Kirkham, M. et al. Ultrastructural identification of uncoated caveolin-independent early endocytic vehicles. J. Cell Biol. 168, 465-476 (2005).

42. Rewatkar, P. V., Parton, R. G., Parekh, H. S. \& Parat, M.-O. Are caveolae a cellular entry route for non-viral therapeutic delivery systems? Adv. Drug Deliv. Rev. 91, 92-108 (2015)

A critical review of studies implicating caveolae in nanoparticle uptake.

43. Richter, T. et al. High-resolution 3D quantitative analysis of caveolar ultrastructure and caveola-cytoskeleton interactions. Traffic $\mathbf{9}$, 893-909 (2008).

44. Chadda, R. et al. Cholesterol-sensitive Cdc42 activation regulates actin polymerization for endocytosis via the GEEC pathway. Traffic $\mathbf{8}$, 702-717 (2007).

45. Pelkmans, L., Kartenbeck, J. \& Helenius, A. Caveolar endocytosis of simian virus 40 reveals a new two-step vesicular-transport pathway to the ER. Nat. Cell Biol. 3, 473-483 (2001).

46. Parton, R. G. \& Howes, M. T. Revisiting caveolin trafficking: the end of the caveosome. J. Cell Biol. 191, 439-441 (2010).

47. Shin, J. S., Gao, Z. \& Abraham, S. N. Involvement of cellular caveolae in bacterial entry into mast cells. Science 289, 785-788 (2000).

48. Rejman, J., Oberle, V., Zuhorn, I. S. \& Hoekstra, D. Size-dependent internalization of particles via the pathways of clathrin- and caveolae-mediated endocytosis. Biochem. J. 377, 159-169 (2004).

49. Iversen, T.-G., Skotland, T. \& Sandvig, K. Endocytosis and intracellular transport of nanoparticles: present knowledge and need for future studies. Nano Today 6, 176-185 (2011).

50. Liebl, D., Qi, X., Zhe, Y., Barnett, T. C. \& Teasdale, R. D. SopB-mediated recruitment of SNX18 facilitates Salmonella typhimurium internalization by the host cell. Front. Cell. Infect. Microbiol. 7, 257 (2017).

51. Aggeler, J. \& Werb, Z. Initial events during phagocytosis by macrophages viewed from outside and inside the cell: membrane-particle interactions and clathrin. J. Cell Biol. 94, 613-623 (1982).

52. Caracciolo, G. et al. Selective targeting capability acquired with a protein corona adsorbed on the surface of 1,2-dioleoyl-3-trimethylammonium propane/DNA nanoparticles. ACS Appl. Mater. Interfaces 5, 13171-13179 (2013)

53. Faria, M. et al. Minimum information reporting in bio-nano experimental literature. Nat. Nanotechnol. 13, 777-785 (2018).

Practical guidelines for studying nanoparticle uptake.

54. Francia, V., Reker-Smit, C., Boel, G. \& Salvati, A. Limits and challenges in using transport inhibitors to characterize how nano-sized drug carriers enter cells. Nanomedicine 14, 1533-1549 (2019).

55. Johnston, A. P. R. Life under the microscope: quantifying live cell interactions to improve nanoscale drug delivery. ACS Sensors 2, 4-9 (2017).

56. Liu, H. \& Johnston, A. P. R. A programmable sensor to probe the internalization of proteins and nanoparticles in live cells. Angew. Chem. Int. Ed. 52, 5744-5748 (2013).
57. Selby, L. I., Aurelio, L., Yuen, D., Graham, B. \& Johnston, A. P. R. Quantifying cellular internalization with a fluorescent click sensor. ACS Sensors 3, 1182-1189 (2018).

58. FitzGerald, L. I. \& Johnston, A. P. R. It's what's on the inside that counts: techniques for investigating the uptake and recycling of nanoparticles and proteins in cells. J. Colloid Interface Sci. 587, 64-78 (2021).

59. Pelkmans, L. et al. Genome-wide analysis of human kinases in clathrin- and caveolae/raft-mediated endocytosis. Nature 436, 78-86 (2005).

60. Sundaramurthy, V. et al. Integration of chemical and RNAi multiparametric profiles identifies triggers of intracellular mycobacterial killing. Cell Host Microbe 13, 129-142 (2013).

61. Jovic, M., Sharma, M., Rahajeng, J. \& Caplan, S. The early endosome: a busy sorting station for proteins at the crossroads. Histol. Histopathol. 25, 99-112 (2010).

62. Kalaidzidis, I. et al. APPL endosomes are not obligatory endocytic intermediates but act as stable cargo-sorting compartments. J. Cell Biol. 211, 123-144 (2015).

63. Zoncu, R. et al. A phosphoinositide switch controls the maturation and signaling properties of APPL endosomes. Cell 136, 1110-1121 (2009).

64. Eyster, C. A. et al. Discovery of new cargo proteins that enter cells through clathrin-independent endocytosis. Traffic 10, 590-599 (2009).

65. Maldonado-Báez, L., Cole, N. B., Krämer, H. \& Donaldson, J. G. Microtubule-dependent endosomal sorting of clathrin-independent cargo by Hook1. J. Cell Biol. 201, 233-247 (2013).

66. Khalil, I. A., Kogure, K., Futaki, S. \& Harashima, H. High density of octaarginine stimulates macropinocytosis leading to efficient intracellular trafficking for gene expression. J. Biol. Chem. 281, 3544-3551 (2006).

67. Selby, L. I., Cortez-Jugo, C. M., Such, G. K. \& Johnston, A. P. R. Nanoescapology: progress toward understanding the endosomal escape of polymeric nanoparticles. WIREs Nanomed. Nanobiotechnol. 9, e1452 (2017). A review of our current understanding of endosomal escape in relation to nanoparticle delivery.

68. Erazo-Oliveras, A. et al. The late endosome and its lipid BMP act as gateways for efficient cytosolic access of the delivery agent dfTAT and its macromolecular cargos. Cell Chem. Biol. 23, 598-607 (2016).

69. Cupic, K. I., Rennick, J. J., Johnston, A. P. \& Such, G. K. Controlling endosomal escape using nanoparticle composition: current progress and future perspectives. Nanomedicine 14, 215-223 (2019).

70. Smith, S. A., Selby, L. I., Johnston, A. P. R. \& Such, G. K. The endosomal escape of nanoparticles: toward more efficient cellular delivery. Bioconjug. Chem. 30, 263-272 (2019).

71. Weigert, R. Imaging the dynamics of endocytosis in live mammalian tissues. Cold Spring Harb. Perspect. Biol. 6, a017012 (2014).

72. Hinze, C. \& Boucrot, E. Endocytosis in proliferating, quiescent and terminally differentiated cells. J. Cell Sci. 131, jcs216804 (2018).

73. Masedunskas, A., Porat-Shliom, N., Rechache, K., Aye, M.-P. \& Weigert, R. Intravital microscopy reveals differences in the kinetics of endocytic pathways between cell cultures and live animals. Cells $\mathbf{1}$, 1121-1132 (2012).

74. Bhirde, A. A. et al. Targeted therapeutic nanotubes influence the viscoelasticity of cancer cells to overcome drug resistance. ACS Nano $\mathbf{8}$, 4177-4189 (2014)

75. Pinilla-Macua, I., Grassart, A., Duvvuri, U., Watkins, S. C. \& Sorkin, A. EGF receptor signaling, phosphorylation, ubiquitylation and endocytosis in tumors in vivo. elife 6, e31993 (2017).

76. Ebrahim, S. \& Weigert, R. Intravital microscopy in mammalian multicellular organisms. Curr. Opin. Cell Biol. 59, 97-103 (2019). A summary of state-of-the-art methods in intravital microscopy being used to study cell biology in vivo.

77. Fung, K. Y. Y., Fairn, G. D. \& Lee, W. L. Transcellular vesicular transport in epithelial and endothelial cells: challenges and opportunities. Traffic 19, 5-18 (2018).

78. Joseph, S. R. et al. An ex vivo human tumor assay shows distinct patterns of EGFR trafficking in squamous cell carcinoma correlating to therapeutic outcomes. J. Invest. Dermatol. 139, 213-223 (2019). An imaging method to study ligand-induced epidermal growth factor receptor internalization in ex vivo human tumour samples.

79. Hansen, S. H., Sandvig, K. \& van Deurs, B. Molecules internalized by clathrin-independent endocytosis are delivered to endosomes containing transferrin receptors. J. Cell Biol. 123, 89-97 (1993).

80. Carpentier, J.-L. et al. Potassium depletion and hypertonic medium reduce non-coated and clathrin-coated pit formation, as well as endocytosis through these two gates. J. Cell. Physiol. 138, 519-526 (1989).

81. Larkin, J. M., Brown, M. S., Goldstein, J. L. \& Anderson, R. G. W. Depletion of intracellular potassium arrests coated pit formation and receptor-mediated endocytosis in fibroblasts. Cell 33, 273-285 (1983).

82. Daniel, J. A. et al. Phenothiazine-derived antipsychotic drugs inhibit dynamin and clathrin-mediated endocytosis. Traffic 16, 635-654 (2015). 
83. Wang, L. H., Rothberg, K. G. \& Anderson, R. G. W. Mis-assembly of clathrin lattices on endosomes reveals a regulatory switch for coated pit formation. J. Cell Biol. 123, 1107-1117 (1993).

84. Sasso, L., Purdie, L., Grabowska, A., Jones, A. T. \& Alexander, C. Time and cell-dependent effects of endocytosis inhibitors on the internalization of biomolecule markers and nanomaterials. J. Interdiscip. Nanomedicine 3, 67-81 (2018)

85. Chen, C.-L. et al. Inhibitors of clathrin-dependent endocytosis enhance TGF signaling and responses. J. Cell Sci. 122, 1863-1871 (2009).

86. von Kleist, L. et al. Role of the clathrin terminal domain in regulating coated pit dynamics revealed by small molecule inhibition. Cell 146 , 471-484 (2011).

87. Dutta, D., Williamson, C. D., Cole, N. B. \& Donaldson, J. G. Pitstop 2 is a potent inhibitor of clathrin-independent endocytosis. PLOS ONE 7, e45799 (2012)

88. Willox, A. K., Sahraoui, Y. M. E. \& Royle, S. J. Non-specificity of Pitstop 2 in clathrin-mediated endocytosis. Biol. Open 3, 326-331 (2014).

89. Macia, E. et al. Dynasore, a cell-permeable inhibitor of dynamin. Dev. Cell 10, 839-850 (2006).

90. McCluskey, A. et al. Building a better dynasore: the Dyngo compounds potently inhibit dynamin and endocytosis. Traffic 14, 1272-1289 (2013).

91. Park, R. J. et al. Dynamin triple knockout cells reveal off target effects of commonly used dynamin inhibitors. J. Cell Sci. 126, 5305-5312 (2013).

92. Kilsdonk, E. P. C. et al. Cellular cholesterol efflux mediated by cyclodextrins. J. Biol. Chem. 270, 17250-17256 (1995).

93. Hao, M., Mukherjee, S., Sun, Y. \& Maxfield, F. R. Effects of cholestero depletion and increased lipid unsaturation on the properties of endocytic membranes. J. Biol. Chem. 279, 14171-14178 (2004).

94. Bolard, J. How do the polyene macrolide antibiotics affect the cellular membrane properties? Biochim. Biophys. Acta Rev. Biomembr. 864, 257-304 (1986).

95. Rentero, C. et al. Functional implications of plasma membrane condensation for T cell activation. PLoS ONE 3, e2262 (2008).

96. Akiyama, T. et al. Genistein, a specific inhibitor of tyrosine-specific protein kinases. J. Biol. Chem. 262, 5592-5595 (1987)

97. Parton, R. G., Joggerst, B. \& Simons, K. Regulated internalization of caveolae. J. Cell Biol. 127, 1199-1215 (1994).

98. Brenner, S. L. \& Korn, E. D. Substoichiometric concentrations of cytochalasin D inhibit actin polymerization. Additional evidence for an F-actin treadmill. J. Biol. Chem. 254, 9982-9985 (1979).

99. Fujimoto, L. M., Roth, R., Heuser, J. E. \& Schmid, S. L. Actin assembly plays a variable, but not obligatory role in receptor-mediated endocytosis. Traffic 1, 161-171 (2000).

100. Gladhaug, I. P. \& Christoffersen, T. Amiloride inhibits constitutive internalization and increases the surface number of epidermal growth factor receptors in intact rat hepatocytes. J. Cell. Physiol. 143, 188-195 (1990).

101. Kleyman, T. R. \& Cragoe, E. J. Amiloride and its analogs as tools in the study of ion transport. J. Membr. Biol. 105, 1-21 (1988).

102. Henriksen, L., Grandal, M. V., Knudsen, S. L. J., van Deurs, B. \& Grøvdal, L. M. Internalization mechanisms of the epidermal growth factor receptor after activation with different ligands. PLoS ONE 8, e58148 (2013).

103. Ceresa, B. P., Kao, A. W., Santeler, S. R. \& Pessin, J. E. Inhibition of clathrin-mediated endocytosis selectively attenuates specific insulin receptor signal transduction pathways. Mol. Cell. Biol. 18, 3862-3870 (1998).

104. Liu, S.-H., Marks, M. S. \& Brodsky, F. M. A dominant-negative clathrin mutant differentially affects trafficking of molecules with distinct sorting motifs in the class II major histocompatibility complex (MHC) pathway. J. Cell Biol. 140, 1023-1037 (1998).

105. Hill, M. M. et al. PTRF-cavin, a conserved cytoplasmic protein required for caveola formation and function. Cell 132, 113-124 (2008).

106. Liberali, P. et al. The closure of Pak1-dependent macropinosomes requires the phosphorylation of CtBP1/BARS. EMBO J. 27, 970-981 (2008).

107. Kalin, S. et al. Macropinocytotic uptake and infection of human epithelial cells with species B2 adenovirus type 35. J. Virol. 84, 5336-5350 (2010).

108. Licona-Limón, I., Garay-Canales, C. A., Muñoz-Paleta, O. \& Ortega, E. CD13 mediates phagocytosis in human monocytic cells. J. Leukoc. Biol. 98, 85-98 (2015).

109. Gambin, Y. et al. Single-molecule analysis reveals self assembly and nanoscale segregation of two distinct cavin subcomplexes on caveolae. eLife 3, e01434 (2014).

110. Bitsikas, V., Corrêa, I. R. \& Nichols, B. J. Clathrin-independent pathways do not contribute significantly to endocytic flux. eLife 3, e03970 (2014).
111. Arredouani, M. S. et al. MARCO Is the major binding receptor for unopsonized particles and bacteria on human alveolar macrophages. J. Immunol. 175, 6058-6064 (2005).

112. Conner, S. D. \& Schmid, S. L. Regulated portals of entry into the cell. Nature 422, 37-44 (2003).

113. King, J. S. \& Kay, R. R. The origins and evolution of macropinocytosis. Philos. Trans. R. Soc. B 374, 20180158 (2019).

114. Yuan, M. et al. Enhanced human enterovirus 71 infection by endocytosis inhibitors reveals multiple entry pathways by enterovirus causing hand-foot-and-mouth diseases. Virol. J. 15, 1 (2018).

115. Volonte, D. et al. Caveolin-1 promotes the tumor suppressor properties of oncogene-induced cellular senescence. J. Biol. Chem. 293, 1794-1809 (2018)

116. Yang, C.-P. H., Galbiati, F., Volonté, D., Horwitz, S. B. \& Lisanti, M. P. Upregulation of caveolin-1 and caveolae organelles in Taxol-resistant A549 cells. FEBS Lett. 439, 368-372 (1998).

117. Qhattal, H. S. S. \& Liu, X. Characterization of CD44-mediated cancer cell uptake and intracellular distribution of hyaluronan-grafted liposomes. Mol. Pharm. 8, 1233-1246 (2011).

118. Yoon, Y.-K. et al. KRAS mutant lung cancer cells are differentially responsive to MEK inhibitor due to AKT or STAT3 activation: implication for combinatorial approach. Mol. Carcinog. 49, 353-362 (2010).

119. Yang, Y. et al. Endophilin A1 regulates dendritic spine morphogenesis and stability through interaction with p140Cap. Cell Res. 25, 496-516 (2015).

120. Torrino, S. et al. EHD2 is a mechanotransducer connecting caveolae dynamics with gene transcription. J. Cell Biol. 217, 4092-4105 (2018).

121. Aït-Slimane, T., Galmes, R., Trugnan, G. \& Maurice, M. Basolateral internalization of GPI-anchored proteins occurs via a clathrin-independent flotillin-dependent pathway in polarized hepatic cells. Mol. Biol. Cell 20, 3792-3800 (2009).

122. Zhang, J. et al. Distinct functions of endophilin isoforms in synaptic vesicle endocytosis. Neural Plast. 2015, 371496 (2015).

123. Moore, R. H. et al. Ligand-stimulated $\beta_{2}$-adrenergic receptor internalization via the constitutive endocytic pathway into rab5-containing endosomes. J. Cell Sci. 108, 2983-2991 (1995).

124. Nonnenmacher, M. \& Weber, T. Adeno-associated virus 2 infection requires endocytosis through the CLIC/GEEC pathway. Cell Host Microbe 10, 563-576 (2011).

125. Chen, S.-L. et al. Endophilin-A2-mediated endocytic pathway is critical for enterovirus 71 entry into caco-2 cells. Emerg. Microbes Infect. 8, 773-786 (2019).

126. Mirre, C., Monlauzeur, L., Garcia, M., Delgrossi, M. H. \& Le Bivic, A. Detergent-resistant membrane microdomains from Caco-2 cells do not contain caveolin. Am. J. Physiol. Cell Physiol. 271, C887-C894 (1996).

127. Zachos, N. C., Alamelumangpuram, B., Lee, L. J., Wang, P. \& Kovbasnjuk, O. Carbachol-mediated endocytosis of NHE3 involves a clathrin-independent mechanism requiring lipid rafts and $\mathrm{Cdc} 42$. Cell. Physiol. Biochem. 33, 869-881 (2014).

\section{Acknowledgements}

This work was supported by the Australian Research Council (Centre of Excellence in Convergent Bio-Nano Science and Technology CE140100036-A.P.R.J. and R.G.P.) and National Health and Medical Research Council of Australia Fellowships (A.P.R.J., APP1141551, R.G.P., APP1156489). J.J.R. was supported by an Australian Government Research Training Program Scholarship. We are grateful to G. Griffiths, E. Boucrot and F. Simpson for insightful comments and thank the members of the Parton and Johnston groups and Centre of Excellence collaborators for discussion.

\section{Competing interests}

The authors declare no competing interests.

\section{Additional information}

Correspondence should be addressed to A.P.R.J. or R.G.P.

Peer review information Nature Nanotechnology thanks the anonymous reviewers for their contribution to the peer review of this work.

Reprints and permissions information is available at www.nature.com/reprints.

Publisher's note Springer Nature remains neutral with regard to jurisdictional claims in published maps and institutional affiliations.

() Springer Nature Limited 2021 